\title{
Validación Argentina de la Serie 19 del Sistema Internacional de Imágenes Afectivas (IAPS)
}

\section{Argentinian validation of set 19 of the International Affective Picture System (IAPS)}

\author{
María Evangelina Estrada * ${ }^{1}$, Anna Teresa Rovella ${ }^{1}$, María Claudia Brusasca ${ }^{1}$, Jorge Leandro Leporati ${ }^{1}$ \\ 1 - Laboratorio de Investigaciones en Ciencias del Comportamiento (LICIC), Facultad de Psicología, Universidad Nacional de San Luis, Argentina.
}

\section{Resumen}

El objetivo de este trabajo fue proporcionar los datos normativos argentinos de la serie 19 del Sistema Internacional de Imágenes Afectivas (IAPS) de Lang (Lang, Bradley, \& Cuthbert, 1997) a fin de contribuir con su validación transcultural. Los participantes fueron 125 estudiantes de la Universidad Nacional de San Luis (67\% mujeres y 33\% varones) que evaluaron las imágenes en las dimensiones emocionales de valencia, activación y dominancia en el Maniquí de Autorreporte (Self-Assessment Manikin - SAM). Los resultados muestran una distribución de las imágenes en el espacio afectivo en forma de boomerang similar a lo encontrado en Estados Unidos (Lang, Bradley, \& Cuthbert, 2008). No se identificaron diferencias en las dimensiones emocionales de valencia y activación, pero sí en dominancia respecto a los datos obtenidos por Lang, Bradley y $\mathrm{Cu}$ thbert (2008). Esta investigación confirma que el Sistema Internacional de Imágenes Afectivas (IAPS) induce estados afectivos similares en diferentes poblaciones y que sus estímulos afectivos son entendidos de manera similar entre argentinos y estadounidenses.

Palabras clave: emoción, Sistema Internacional de Imágenes Afectivas (IAPS), valencia, activación, dominancia

\begin{abstract}
The aim of this study was to report the Argentinian norms of set 19 of the International Affective Picture System (IAPS) developed by Lang (Lang, Bradley, \& Cuthbert, 1997 ) in order to contribute to the instrument's cross-cultural validation. The participants were 125 students from Universidad Nacional de San Luis (San Luis National University) (67\% women and $33 \%$ men) who assessed the images in the emotional dimensions of valence, arousal and dominance through the Self-Assessment Manikin (SAM). The results showed a distribution of the pictures within the affective space in a boomerang form, which is similar to the American sample (Lang, Bradley, \& Cuthbert, 2008). No differences in the emotional dimensions of valence and arousal were identified, but they were found in dominance regarding the data obtained by Lang et al. (2008). This study confirms that the International Affective Picture System (IAPS) induces similar affective states in different populations and its affective stimulus are understood similarly by Argentinian and American subjects.
\end{abstract}

Key words: emotion, International Affective Picture System (IAPS), valence, arousal, dominance

\footnotetext{
* Correspondencia a: María Evangelina Estrada. Dirección Postal: Falucho 1087, Planta Baja, San Luis, Argentina. Teléfono: +54 92664302410. 


\section{Introducción}

Las emociones son un sistema de procesamiento de información prioritaria para la supervivencia y la adaptación al medio y es por ello que se convierten en el proceso que coordina a los restantes recursos psicológicos necesarios para dar la respuesta más rápida y puntual ante una situación concreta (Fernández-Abascal, García-Rodríguez, Jiménez-Sánchez, Martín-Díaz, \& Domínguez-Sánchez, 2010).

La evaluación de las emociones y de las dimensiones que las conforman es uno de los principales objetivos de la investigación actual en Psicología (Bradley, 2009). En este sentido se hace necesario contar con un instrumento que pueda ser utilizado en el estudio científico de las emociones para inducir estados afectivos de forma fiable, apoyado en un modelo conceptual sólido y que esté adaptado a las características del contexto en el que se presenta. La aproximación teórica propuesta por Lang (1995) define a las emociones como predisposiciones para la acción que surgen a partir de la activación de circuitos cerebrales ante estímulos relevantes para el individuo y plantea una organización jerárquica de la estructura emocional distinguiendo tres niveles: los patrones específicos de la respuesta emocional, los programas emocionales que dan origen a estereotipias de respuestas emocionales ante diversas situaciones y las tres dimensiones, valencia, activación y dominancia, evaluadas por el Sistema Internacional de Imágenes Afectivas (IAPS). La dimensión de valencia (agradable-desagradable) es la que ejerce la principal influencia en la organización jerárquica de las emociones debido a la existencia en el cerebro de dos sistemas motivacionales primarios: el apetitivo (conducta consumatoria, sexual o de crianza) y el defensivo (conducta de protección, escape o evitación). La dimensión de activación hace referencia al nivel de energía invertido en la emoción y representa la activación metabólica y neural de cualquiera de los dos sistemas, tanto el apetitivo como el defensivo. La dominancia es la dimensión que explica el grado de control percibido sobre la respuesta emocional e implica la interrupción o continuidad de la respuesta conductual (Bradley, Codispoti, Cuthbert, \& Lang, 2001). Las dimensiones emocionales son evaluadas mediante un sistema de autoinforme desarrollado por Bradley y Lang (1994), el Maniquí de Autoevaluación (Self-Assessment Manikin - SAM).

En relación a la configuración emocional, Lang postula que tiene una forma de "boomerang", o cuarto de luna (Bradley \& Lang, 2007). Esta forma viene determinada porque no hay ocurrencia de situaciones extremas en valencia afectiva (tanto positiva como negativa) que presenten una baja activación; del mismo modo, cuando hay una extrema activación las situaciones no pueden ser neutras en valencia afectiva.

En Argentina existen escasas investigaciones respecto a la medición de las dimensiones de la emoción mediante el uso de imágenes afectivas (Estrada, Rovella, \& Brusasca, 2015; Martínez-Núñez et al., 2013). Por esta razón, el objetivo de esta investigación fue adaptar a la población nacional una de las veinte series de imágenes, serie 19, que componen el Sistema Internacional de Imágenes Afectivas (IAPS), y de esta manera contribuir al proceso de adaptación de dicho instrumento a la población argentina.

\section{Método}

Participantes

El estudio se llevó a cabo con una muestra compuesta por un total de 125 sujetos, de ambos sexos, estudiantes de la Universidad Nacional de San Luis, con edades comprendidas entre 17 y 52 
años de edad (media de edad: 21,6 y desviación estándar: 5,13 ). Se controló que la proporción de hombres y mujeres fuera de 1:2 respectivamente, siguiendo las pautas propuestas por Lang et al. (2008).

\section{Instrumentos}

Sistema Internacional de Imágenes Afectivas (IAPS; Lang et al., 1997). Se utilizaron 59 imágenes a color, en formato digitalizado, pertenecientes a la serie 19 del Sistema Internacional de Imágenes Afectivas (IAPS), presentadas en diapositivas a través de una presentación de PowerPoint y un proyector Video Beam en espacios cerrados. Las imágenes y fotografías afectivas que se utilizan tienen la característica de ser estímulos perceptuales complejos y altamente simbólicos.

\section{Maniquí de Autoevaluación (Self-Assess-} ment Manikin - SAM; Bradley \& Lang, 1994). Se empleó para realizar la evaluación de las imágenes afectivas en cada una de las dimensiones. Está conformado por tres escalas pictográficas, cada una con cinco dibujos humanoides, diagramadas a lo largo de un continuo que representan cada una de las tres dimensiones de la emoción: valencia (agradable-desagradable), activación (relajado-activado) y dominancia (dominante-dominado). Las figuras que representan la dimensión de valencia están conformadas por rostros que varían en intensidad de felicidad hasta tristeza, las que representan la dimensión de activación varían en intensidad de activación hasta calma; y finalmente las que representan la dimensión de dominancia varían en el tamaño para indicar el nivel de control percibido sobre la emoción.

La calificación del instrumento se realiza señalando con una " $X$ " alguna de las cinco figuras o los espacios que hay entre ellas en cada una de las dimensiones, lo cual resulta en una puntuación que va de 1 a 9. Se utilizó el cuadernillo construido para la adaptación española (Vila-Castellar et al., 2001), conformado por seis páginas con diez líneas por página, en cada línea aparecen las tres dimensiones organizadas de izquierda a derecha y en el margen izquierdo el número de la diapositiva que cada persona debe evaluar.

\section{Procedimiento}

En primer lugar se realizó una convocatoria a los alumnos de las distintas facultades de la Universidad Nacional de San Luis. Se les solicitó su colaboración voluntaria haciendo hincapié en que su participación sería anónima. Quienes estuvieron interesados en participar registraron sus datos personales en una planilla de registro en la cual se encontraban detallados los días y horarios en los cuales se suministrarían los cuestionarios.

La aplicación se realizó en un encuentro en forma grupal, en grupos no mayores de 20 sujetos, en los días y horarios previamente estipulados en la planilla. El lugar donde se llevó a cabo fue la Cámara Gesell ubicada en el Cuarto Bloque de la Universidad Nacional de San Luis.

Previamente se les informó a los participantes sobre las características y los objetivos de la investigación. Se presentó el consentimiento informado, se les pidió que lo leyeran con detenimiento para ser firmado posteriormente.

En primer lugar, se administró el Sistema Internacional de Imágenes Afectivas (IAPS). La presentación de las imágenes y las instrucciones se realizaron siguiendo estrictamente el protocolo desarrollado por Lang et al. (2008). Seguidamente, se entregó el Maniquí de Autoevaluación para la evaluación de las imágenes y posteriormente se proyectaron las instrucciones del Sistema Inter- 
nacional de Imágenes Afectivas (IAPS) a través de un PowerPoint en donde se mostró el proceso a seguir y una prueba piloto con tres imágenes.

\section{Resultados}

A continuación se presentan los resultados de la validación de la serie 19 del Sistema Internacional de Imágenes Afectivas (IAPS) en la población argentina y las comparaciones entre los resultados obtenidos en Argentina y en Estados Unidos.

Valores normativos y espacio bidimensional afectivo

Estadísticos descriptivos: En la Tabla 1 se presentan las medias y las desviaciones estándar de las evaluaciones en las tres dimensiones emocionales (valencia, activación y dominancia), para cada una de las 59 imágenes de la serie 19 del Sistema Internacional de Imágenes Afectivas (IAPS), organizadas según el número internacional de clasificación (Lang et al., 2008). Estas medidas se presentan para la totalidad de participantes. Dichos datos corresponden a los valores normativos de la validación argentina.

Espacio bidimensional afectivo: En la Figura 1 se representa la distribución de las 59 imágenes en el espacio bidimensional formado por las dimensiones de valencia afectiva (eje vertical) y activación (eje horizontal). La valencia, eje vertical, inicia en el número 1 (altamente desagradable), 5 (neutra) y 9 (altamente agradable). La activación, eje horizontal, inicia también en 1 (completa calma), 5 (activación moderada) y 9 (alta activación). En el gráfico la distribución adopta la forma de boomerang, con dos brazos que salen desde una base afectivamente neutra y calmada hacia los dos extremos activadores agradable y desagradable. Esta forma característica refleja el hecho de que (1) las imágenes neutras suelen ser evaluadas como poco activadoras, y (2) que a medida que se extreman las puntuaciones en la dimensión de valencia afectiva, aumentan las puntuaciones en activación -es decir, las imágenes evaluadas con un mayor nivel de agrado y de desagrado tienden también a obtener las puntuaciones más elevadas en la dimensión de activación. En cuanto al brazo que se extiende hacia el polo desagradable tiene una mayor inclinación, y una menor dispersión, que el que se extiende hacia el polo agradable. Esto sugiere que lo muy desagradable va acompañado de un alto nivel de activación y apoya la idea de que para la supervivencia es básica una rápida activación del sistema defensivo. Estos resultados coinciden con los datos de la muestra estadounidense (Lang et al., 2008).

Las imágenes, representadas en la Figura 1, que se puntuaron como desagradables o negativas están asociadas a objetos rotos, en mal estado o dañinos para la salud, y a operaciones y muerte. Por otra parte, las imágenes que se puntuaron como agradables o positivas están relacionadas a la naturaleza, al hogar y a niños.

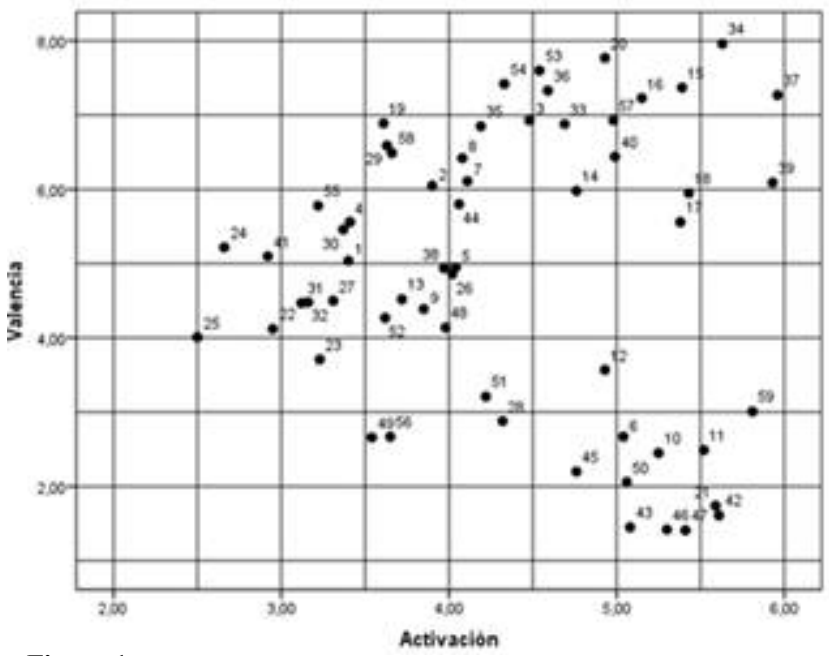

Figura 1

Distribución de las 59 imágenes de la serie 19 del Sistema Internacional de Imágenes Afectivas (IAPS) en el espacio bidimensional para todos los participantes. 
Tabla 1

Estadísticos descriptivos (medias y desviaciones estándar) de las dimensiones emocionales - valencia, activación y dominancia - de cada una de las 59 imágenes de la serie 19 del Sistema Internacional de Imágenes Afectivas (IAPS) para la muestra total.

\begin{tabular}{|c|c|c|c|c|c|c|c|}
\hline \multirow[t]{2}{*}{$\mathbf{N}^{\circ}$} & \multirow[t]{2}{*}{ Descripción } & \multicolumn{2}{|c|}{ Valencia } & \multicolumn{2}{|c|}{ Activación } & \multicolumn{2}{|c|}{ Dominancia } \\
\hline & & $\mathbf{M}$ & DE & $\mathbf{M}$ & DE & $\mathbf{M}$ & DE \\
\hline 1271 & Cucarachas & 2,67 & $(1,58)$ & 3,65 & $(2,00)$ & 5,07 & $(2,53)$ \\
\hline 1595 & Poni & 6,93 & $(1,97)$ & 4,98 & $(2,36)$ & 6,21 & $(2,19)$ \\
\hline 1605 & Mariposa & 6,49 & $(1,86)$ & 3,66 & $(2,06)$ & 6,27 & $(2,48)$ \\
\hline 1820 & Cocodrilo & 3,01 & $(2,23)$ & 5,81 & $(2,48)$ & 4,41 & $(2,40)$ \\
\hline 2115 & Piercing & 3,21 & $(2,21)$ & 4,22 & $(2,43)$ & 5,15 & $(2,41)$ \\
\hline 2122 & Lengua afuera & 4,27 & $(2,33)$ & 3,62 & $(2,05)$ & 5,64 & $(2,47)$ \\
\hline 2151 & Padre/Niña & 7,60 & $(1,58)$ & 4,54 & $(2,55)$ & 6,90 & $(2,11)$ \\
\hline 2274 & Niños & 7,42 & $(1,58)$ & 4,33 & $(2,39)$ & 6,86 & $(2,08)$ \\
\hline 2302 & Niña/Cámara & 5,78 & $(1,91)$ & 3,22 & $(1,90)$ & 6,21 & $(2,25)$ \\
\hline 2308 & Niña/Maquillaje & 5,04 & $(2,02)$ & 3,40 & $(1,93)$ & 5,93 & $(2,29)$ \\
\hline 2377 & Leyendo & 6,05 & $(1,81)$ & 3,90 & $(2,02)$ & 6,45 & $(2,10)$ \\
\hline 2382 & Artista & 6,93 & $(1,58)$ & 4,48 & $(2,21)$ & 6,61 & $(2,26)$ \\
\hline 2390 & Pareja & 5,56 & $(1,82)$ & 3,41 & $(2,06)$ & 6,15 & $(2,26)$ \\
\hline 2392 & Hombre con pescado & 4,95 & $(2,32)$ & 4,04 & $(2,18)$ & 5,80 & $(2,42)$ \\
\hline 2457 & Niño llorando & 2,67 & $(1,79)$ & 5,04 & $(2,47)$ & 4,51 & $(2,20)$ \\
\hline 2489 & Músico & 6,11 & $(1,79)$ & 4,11 & $(2,25)$ & 6,25 & $(2,16)$ \\
\hline 2511 & Anciana & 6,42 & $(1,95)$ & 4,08 & $(2,31)$ & 6,43 & $(2,06)$ \\
\hline 2525 & Mujeres & 4,39 & $(1,93)$ & 3,85 & $(2,17)$ & 5,48 & $(2,46)$ \\
\hline 3019 & Órganos & 2,45 & $(1,94)$ & 5,25 & $(2,77)$ & 4,01 & $(2,56)$ \\
\hline 3212 & Operación & 2,49 & $(1,98)$ & 5,52 & $(2,75)$ & 3,97 & $(2,36)$ \\
\hline 3310 & Incubadora & 3,57 & $(2,47)$ & 4,93 & $(2,40)$ & 4,76 & $(2,41)$ \\
\hline 4008 & Mujer erótica & 4,52 & $(2,55)$ & 3,72 & $(2,50)$ & 5,02 & $(2,80)$ \\
\hline 4525 & Hombre atractivo & 5,98 & $(2,27)$ & 4,76 & $(2,55)$ & 6,07 & $(2,34)$ \\
\hline 4628 & Romance & 7,37 & $(1,53)$ & 5,39 & $(2,44)$ & 6,85 & $(2,09)$ \\
\hline 4628 & Boda & 7,23 & $(1,87)$ & 5,15 & $(2,56)$ & 6,85 & $(2,09)$ \\
\hline 4692 & Pareja erótica I & 5,56 & $(2,34)$ & 5,38 & $(2,58)$ & 5,37 & $(2,48)$ \\
\hline 4693 & Pareja erótica II & 5,95 & $(2,45)$ & 5,43 & $(2,48)$ & 5,43 & $(2,53)$ \\
\hline 5726 & Granos & 6,89 & $(1,84)$ & 3,61 & $(2,31)$ & 6,85 & $(2,31)$ \\
\hline 5829 & Puesta de sol & 7,77 & $(1,36)$ & 4,93 & $(2,52)$ & 6,94 & $(1,95)$ \\
\hline 6520 & Ataque & 1,74 & $(1,38)$ & 5,56 & $(2,80)$ & 3,98 & $(2,47)$ \\
\hline 6837 & Policía & 4,12 & $(1,46)$ & 2,95 & $(1,96)$ & 5,67 & $(2,55)$ \\
\hline 7013 & Foco & 3,71 & $(1,56)$ & 3,23 & $(1,94)$ & 5,11 & $(2,70)$ \\
\hline 7026 & Mesa de picnic & 5,22 & $(1,61)$ & 2,66 & $(1,75)$ & 6,24 & $(2,57)$ \\
\hline 7032 & Zapatillas & 4,01 & $(1,60)$ & 2,50 & $(1,68)$ & 5,72 & $(2,55)$ \\
\hline 7033 & Tren & 4,86 & $(1,68)$ & 4,02 & $(2,24)$ & 6,15 & $(2,25)$ \\
\hline
\end{tabular}




\begin{tabular}{llllllll}
7077 & Hornalla & 4,50 & $(1,39)$ & 3,31 & $(2,10)$ & 5,87 & $(2,43)$ \\
7135 & Daño del auto & 2,88 & $(1,59)$ & 4,32 & $(2,41)$ & 5,31 & $(2,31)$ \\
7165 & Baño & 6,59 & $(1,53)$ & 3,63 & $(2,24)$ & 6,98 & $(1,85)$ \\
7255 & Galleta & 5,46 & $(1,62)$ & 3,37 & $(1,79)$ & 6,15 & $(2,30)$ \\
7287 & Tomate & 4,47 & $(1,84)$ & 3,12 & $(1,82)$ & 5,85 & $(2,56)$ \\
7354 & Ajo & 4,48 & $(2,19)$ & 3,16 & $(1,94)$ & 5,77 & $(2,63)$ \\
7440 & Parrilla & 6,88 & $(1,82)$ & 4,69 & $(2,35)$ & 6,28 & $(2,24)$ \\
7492 & Transbordador & 7,96 & $(1,35)$ & 5,63 & $(2,45)$ & 6,87 & $(1,99)$ \\
7509 & Pincel & 6,85 & $(1,96)$ & 4,19 & $(2,36)$ & 6,59 & $(2,19)$ \\
7530 & Casa & 7,33 & $(1,56)$ & 4,59 & $(2,41)$ & 6,96 & $(2,07)$ \\
7660 & Multitud & 7,27 & $(1,74)$ & 5,96 & $(2,25)$ & 6,02 & $(2,43)$ \\
8121 & Atleta & 4,94 & $(1,84)$ & 3,97 & $(2,05)$ & 6,00 & $(2,34)$ \\
8206 & Surfistas & 6,09 & $(1,92)$ & 5,93 & $(2,29)$ & 6,44 & $(1,69)$ \\
8208 & Surfista & 6,44 & $(1,69)$ & 4,99 & $(2,33)$ & 6,17 & $(2,26)$ \\
8312 & Golf & 5,10 & $(1,85)$ & 2,92 & $(1,83)$ & 5,92 & $(2,78)$ \\
9163 & Soldados & 1,61 & $(1,37)$ & 5,61 & $(2,94)$ & 3,60 & $(2,35)$ \\
9185 & Perro muerto & 1,45 & $(0,90)$ & 5,08 & $(2,90)$ & 3,32 & $(2,52)$ \\
9260 & Manos & 5,80 & $(2,00)$ & 4,06 & $(2,13)$ & 6,02 & $(2,28)$ \\
9321 & Vómito & 2,20 & $(1,87)$ & 4,76 & $(2,75)$ & 3,76 & $(2,57)$ \\
9412 & Hombre muerto & 1,42 & $(0,82)$ & 5,30 & $(2,88)$ & 3,38 & $(2,34)$ \\
9413 & Ahorcados & 1,41 & $(0,99)$ & 5,41 & $(2,87)$ & 3,28 & $(2,42)$ \\
9468 & Grafitti & 4,14 & $(2,03)$ & 3,98 & $(2,42)$ & 5,32 & $(2,50)$ \\
9832 & Cigarrillos & 2,66 & $(1,83)$ & 3,54 & $(3,24)$ & 5,24 & $(2,80)$ \\
9904 & Accidente de auto & 2,06 & $(1,50)$ & 5,06 & $(2,66)$ & 4,26 & $(2,43)$ \\
\hline
\end{tabular}

Comparaciones entre la evaluación argentina y la estadounidense

En la Tabla 2 se presentan los valores obtenidos por ambas muestras en relación a la serie 19 del Sistema Internacional de Imágenes Afectivas (IAPS) con el propósito de indagar las variaciones obtenidas en la Serie 19 del Sistema Internacional de Imágenes Afectivas (IAPS) tanto en los puntajes obtenidos en la muestra estadounidense como en los obtenidos en la muestra argentina. Tales puntajes, diferencias de rangos promedios, fueron estimados a través de la prueba de comparación de medias para muestras independientes (prueba $t$ de Student) y el nivel de significancia bilateral. Se tomaron como criterios de agrupación las variables valencia, activación y dominancia.
No se observan diferencias significativas entre la muestra estadounidense y la muestra argentina en los puntajes de las dimensiones valencia y activación (Lang et al., 2008).

En relación a la dimensión dominancia, se encontraron diferencias significativas $(p=.021)$, siendo mayor el grado de control percibido en la muestra argentina. Estos resultados evidencian que los estímulos afectivos del Sistema Internacional de Imágenes Afectivas (IAPS) son entendidos de manera similar entre argentinos $\mathrm{y}$ estadounidenses, y que la diferencia radica en la regulación emocional. 
Tabla 2

Comparación de medias de las evaluaciones argentinas y estadounidenses para la serie 19 del Sistema Internacional de Imágenes Afectivas (IAPS).

\begin{tabular}{lllccc}
\hline & N & Media & Prueba $\boldsymbol{t}$ de Student & $\begin{array}{c}\text { Significación } \\
\text { bilateral }\end{array}$ \\
\hline Valencia & EEUU & 59 & 5.0508 & .15373 & .637 \\
\multirow{4}{*}{ Activación } & Arg & 59 & 4.8971 & & \\
& EEUU & 59 & 4.6827 & .00000 & 1 \\
Dominancia & Arg & 59 & 4.6827 & & .021 \\
& EEUU & 59 & 5.2103 & -.42831 & \\
& Arg & 59 & 5.6386 & & \\
\hline
\end{tabular}

\section{Discusión}

En las últimas décadas se ha producido una revitalización en el interés en el estudio científico de la emoción, lo que ha implicado una necesidad creciente por encontrar medidas fiables y válidas de los procesos afectivos. En este sentido, el Sistema Internacional de Imágenes Afectivas (IAPS) ha demostrado ser un instrumento con una excelente bondad psicométrica, debido a que reúne todos los requisitos necesarios propuestos para cualquier método de inducción de estados emocionales (Moltó et al., 2013).

En este trabajo se llevó a cabo la validación de la serie 19 del Sistema Internacional de Imágenes Afectivas (IAPS) a fin de obtener los valores normativos- en valencia, activación y dominancia- para la muestra argentina y compararlos con la muestra estadounidense. Además, se pretende contribuir al proceso de adaptación de dicho instrumento a la población argentina, de forma que los investigadores de nuestro país puedan disponer de unos valores normativos adecuados a su contexto sociocultural para seleccionar y controlar los estímulos emocionales que utilicen en sus experimentos.

Los resultados obtenidos en esta investigación permiten afirmar que la validación de la serie 19 del Sistema Internacional de Imágenes Afectivas (IAPS) se ha realizado adecuadamente, debido a que apoyan de manera consistente las conclusiones aportadas por Lang en la muestra estadounidense (Lang et al., 2008).

La representación de las imágenes en el espacio bidimensional afectivo, definido por las dimensiones de valencia y activación, confirma la forma de boomerang encontrada en las evaluaciones de Lang. Esta dispersión característica de las imágenes se debe, por un lado, a que los estímulos se distribuyen en la dimensión de valencia desde un punto central neutro y calmado hacia sus polos extremos (agradable y desagradable) a medida que aumenta progresivamente el nivel de activación y, por otro, a la ausencia de imágenes neutras muy activadoras.

No se encontraron diferencias en las dimensiones de valencia y activación entre la evaluación argentina y estadounidense, pero sí en la dimensión dominancia. Este resultado no sólo demuestra la utilidad de las imágenes afectivas para provocar respuestas en el nivel expresivo-evaluativo de la emoción, que resultaron muy similares en ambas muestras, y por lo tanto en distintos países y culturas, sino que permiten la replicación de los estudios y la corroboración de los resultados experimentales en distintos laboratorios. No obstante, y a pesar de que las imágenes afectivas pa- 
recen ser entendidas de manera similar por argentinos y estadounidenses, se encontraron algunas diferencias culturales como queda evidenciado en las evaluaciones de la dominancia. Esto quiere decir que los argentinos perciben las imágenes afectivas con un mayor nivel de control, tienden a dominarlas más, que los estadounidenses.

Las limitaciones de este trabajo de investigación radican en la composición de la muestra, ya que se evaluaron sólo sujetos sanluiseños, no se incluyeron participantes de otras provincias.

Para futuras investigaciones se sugiere incluir medidas fisiológicas para estudiar las emociones, ya que éstas son matrices de respuesta multisistémicas, y sería beneficioso medirlas más ampliamente de lo que habitualmente se hace. Además de evaluar las imágenes en el Maniquí de Autoevaluación (SAM), o complementariamente a éste, sería importante medir la respuesta fisiológica emocional provocada por los estímulos pictográficos afectivos. Por otra parte, también sería recomendable trabajar en una versión local del Sistema Internacional de Imágenes Afectivas (IAPS), a partir de la creación de una base de estímulos pictóricos afectivos similares a los de este instrumento pero que incluya las particularidades y peculiaridades de nuestro contexto y que contribuya a la actualización de algunos temas o contenidos representados en las imágenes.

\section{Referencias}

Bradley, M. (2009). Natural selective attention: Orienting and emotion. Psychophysiology, 46(1), 1-11. doi: 10.1111/j.1469-8986.2008.00702.x

Bradley, M., Codispoti, M., Cuthbert, B., \& Lang, P. (2001). Emotion and motivation I: Defensive and appetitive reactions in picture processing.
Emotion, 1(3), 276-298. doi: 10.1037/15283542.1.3.276

Bradley, M., \& Lang, P. (1994). Measuring emotion: The self-assessment manikin and the semantic differential. Journal of Behavior Therapy and Experimental Psychiatry, 25(1), 49-59. doi: 10.1016/0005-7916(94)90063-9

Bradley, M., \& Lang. P. (2007). Emotion and motivation. En J. T. Cacciopo, L. G. Tassinary \& G. Berntson (Eds.), Handbook of Psychophysiology (pp. 581-607). New York: Cambridge University Press.

Estrada, M. E., Rovella, A. T., \& Brusasca, M. C. (2015). Datos preliminares de la validación de la serie 19 del Sistema Internacional de Imágenes Afectivas (IAPS) de Lang en una muestra de estudiantes universitarios de San Luis. En V. Martínez \& S. Luquez (Eds.), Memorias del I Congreso Nacional de Psicología "Psicología, Formación y Compromiso social" (pp. 119120). San Luis: Nueva Editorial Universitaria.

Fernández-Abascal, E., García-Rodríguez, B., Jiménez-Sánchez, M., Martín-Díaz, M., \& Domínguez-Sánchez, F. (2010). Psicología de la emoción. Madrid: Editorial Universitaria Ramón Areces.

Lang, P. (1995). The emotion probe. Studies of motivation and attention. American Psychologist, 50(5), 372-385. doi: 10.1037/0003066X.50.5.372

Lang, P., Bradley, M., \& Cuthbert, B. (1990). Emotion, attention, and the startle reflex. Psychological Review, 97(3), 377-398. doi: 10.1037/0033295x.97.3.377

Lang, P., Bradley, M., \& Cuthbert, B. (1997). International Affective Picture System (IAPS): Tech- 
nical manual and affective ratings. Bethesda, MD: NIMH Center for the Study of Emotion and Attention.

Lang, P., Bradley, M., \& Cuthbert, B. (2008). International Affective Picture System (IAPS): Affective ratings of pictures and instruction manual. Technical Report A-8. Florida: Center for Research in Psychophysiology.

Martínez-Núñez, V., Pitoni, D., Brusasca, C., Rodríguez, M., Rovella, A., García-Quiroga, E., ... Hernández, M. M. (2013). Imágenes afectivas de Lang: Datos preliminares de la validación en una muestra de estudiantes universitarios en San Luis, Argentina. Revista Argentina de Ciencias del Comportamiento. Suplemento (Julio),103-105.

Moltó, J., Segarra, P., Esteller, A., Fonfría, A., Pastor, C., \& Poy, R. (2013). Adaptación española del "International Affective Picture System" (IAPS). Tercera parte. Anales de Psicología, 29(3), 965984. doi: 10.6018/analesps.29.3.153591

Vila-Castellar, J., Sánchez, M., Ramírez-Uclés, I., Fernández-Santaella, M., Cobos-Álvarez, P., Rodríguez-Fernandez, S., ... Moltó, J. (2001). El Sistema Internacional de Imágenes Afectivas (IAPS): Adaptación española. Segunda parte. Revista de Psicología General y Aplicada, 54(4), 635-657. Recuperado de https://dialnet. unirioja.es/servlet/articulo? codigo=2364951 